\title{
Die Kulturverständige
}

ZUHAL COLAK Sich mit Patienten aus anderen Kulturen zu verständigen, ist oft schwierig nicht nur sprachliche Barrieren stehen dabei im Weg. Für eine erfolgreiche Behandlung ist es deshalb umso wichtiger, die Kultur des Patienten zu verstehen, meint Zuhal Colak.

Die junge Physiotherapeutin machte das Thema zu ihrer Bachelorarbeit und stellte fest, dass die Interkulturalität in der Wissenschaft noch nicht angekommen ist.

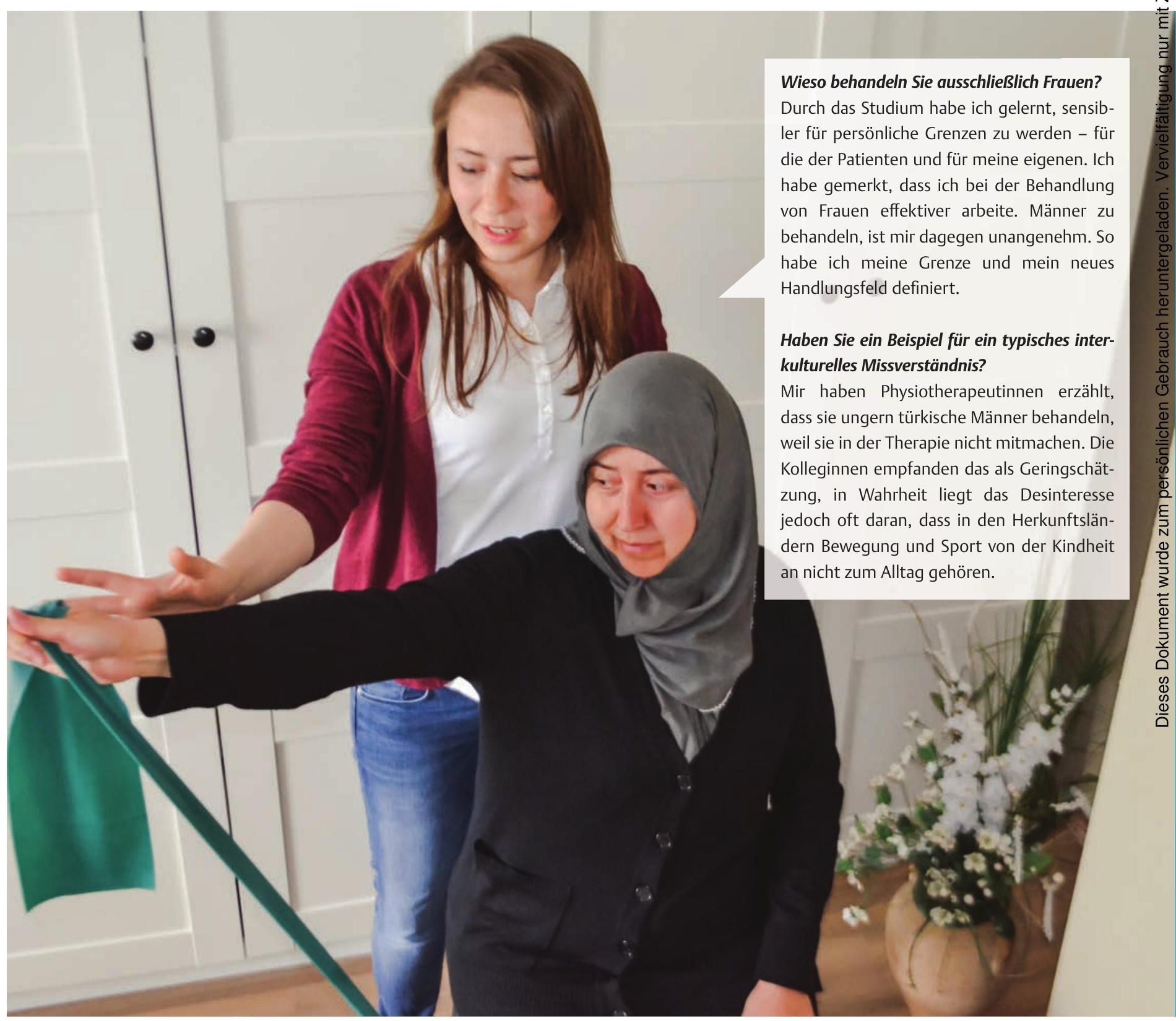




\section{Kultursensibilität im Clinical Reasoning}

\section{Zuhal Colak ...}

... ist 29 Jahre alt und lebt in Rodenberg in Niedersachsen. Ihre Eltern stammen ursprünglich aus der Türkei. Sie selbst ist in Deutschland geboren und aufgewachsen - geprägt von der türkisch-islamischen und der deutsch-christlichen Kultur.

2008 hat sie ihre Physiotherapieausbildung in Bad Lauterberg im Harz beendet. Um später auch im Ausland arbeiten zu können, begann sie im Anschluss das Bachelorstudium Physiotherapie an der HAWK Hildesheim. Ein Auslandssemester verbrachte sie an der Universität Hacettepe in Ankara, wo sie auch nach dem Studium als Physiotherapeutin arbeiten wollte. Aber es kam anders. Sie heiratete, bekam eine Tochter und schloss in Deutschland ihr Studium ab. Seit der Baby-Pause arbeitet Zuhal Colak in einer Praxis in Garbsen nahe Hannover. Ihrer Chefin dort ist sie sehr dankbar, dass diese sie trotz einer Besonderheit eingestellt hat: Zuhal Colak behandelt nur Frauen. In der Zukunft könnte sie sich vorstellen, zudem in die Forschung über Interkulturalität einzusteigen. In ihrer Freizeit liest sie Bücher über fremde Kulturen und verreist gerne. Außerdem ist sie viel in der Natur unterwegs und nimmt zum Abschalten an Zumba- und StepAerobic-Kursen teil.

\section{Die Bachelorarbeit}

In Deutschland leben etwa 16 Millionen Menschen mit einem Migrationshintergrund. Der größte Teil von ihnen kommt aus der Türkei. Die kulturelle Vielfalt der deutschen Gesellschaft bereichert zwar das Zusammenleben der Menschen, gleichzeitig sind die verschiedenen Sprachen und Wertesysteme aber auch eine große Herausforderung für die Gesundheitsversorgung.

Eine Migrationsgeschichte prägt das Leben der Betroffenen in besonderem Maße und wirkt sich oft auf deren Gesundheit aus. Migration bedeutet Entwurzelung und kann mit Stress und Unsicherheiten einhergehen. Häufig haben diese Menschen schlechte Lebensbedingungen, etwa ein geringes Einkommen oder eine schlechte Wohnumgebung. Um die gesundheitlichen Probleme von Migranten erfassen zu können, ist ein tiefes Verständnis für die Biografien, Lebenswelten und sozialen Rahmenbedingungen, in denen sie leben, erforderlich. Diese lassen sich mithilfe des Clinical Reasoning herausfinden.

Doch trotz dieses Ansatzes empfinden viele Physiotherapeuten die Behandlung von türkisch-islamischen Patienten als schwierig. Neben der Sprachbarriere gibt es in der Behandlung häufig kulturelle Differenzen, wie die Rollenverteilung von Männern und Frauen oder das Schamgefühl türkischer Frauen davor, sich auszuziehen.

Scham wahrt die Identität eines Menschen und setzt körperliche und seelische Grenzen. Ein Mensch, der Scham empfindet, erlebt dies als sehr starkes und unter Umständen quälendes Gefühl, das mit psychischen und physischen Reaktionen einhergeht. Schamgrenzen verlaufen bei jeder Kultur anders. Im klinischen Alltag ist Scham jedoch häufig und nahezu unvermeidlich. Diesem Thema wollte Zuhal Colak in ihrer Bachelorarbeit genauer auf den Grund gehen und stellte sich die Frage, ob und wie ein kulturell bedingtes Schamgefühl im physiotherapeutischen Clinical-Reasoning-Prozess in Deutschland berücksichtigt wird. Dazu führte sie eine Literaturrecherche in diversen Datenbanken, Fachzeitschriften und -büchern durch.

\section{Die Ergebnisse}

Zuhal Colak hat herausgefunden und beobachtet, dass...

$>$ es in der deutsch- und englischsprachigen Fachliteratur keine Publikationen zum Thema Schamgefühl, ausgelöst durch Nacktheit, im Kontext einer physiotherapeutischen Behandlung gibt.

> die Physiotherapie hierzulande von der freizügigeren deutschen Kultur geprägt ist und das Sich-Ausziehen bereits für Physiotherapieschüler normal und selbstverständlich ist.

> Patienten mit einem türkisch-islamischen Hintergrund die Therapie hingegen als ungewohnt erleben können, was ihr Selbstbild stört und Scham auslöst.

$>$ der Krankheitsfall aus Sicht des Islam zwar eine besondere Situation darstellt, in der zum Beispiel auch ein Physiotherapeut eine türkisch-islamische Patientin behandeln kann, die Frauen dies aber trotzdem als unangenehm empfinden können und ihre Scham verdrängen.

\section{Das Fazit}

Zusammenfassend kann Zuhal Colak festhalten, dass ...

$>$ es für das Wohlbefinden und den Therapieerfolg förderlich ist, eine angenehme und möglichst schamfreie Atmosphäre für Therapeut und Patient zu schaffen. Dazu kann es notwendig sein, nur gleichgeschlechtliche Behandlungen durchzuführen.

> Physiotherapeuten lernen sollten, kulturelle und migrationsspezifische Aspekte in das Clinical Reasoning einzubinden, um kultursensibel zu behandeln. Dazu gehört, dass sie etwa die Hintergründe kennen und Verhaltensweisen verstehen lernen. Zudem sollten sie ihre Sichtweisen zu kulturellen Gemeinsamkeiten und Unterschieden reflektieren.

$>$ erheblicher Forschungsbedarf zur kultursensiblen Physiotherapie besteht.

Eva Trompetter

$\Rightarrow$ Colak Z. Kultursensibilität im Clinical Reasoning am Beispiel des Schamgefühls. Bachelorarbeit an der Hochschule für angewandte Wissenschaft und Kunst Hildesheim; 2012 